\title{
POLÉMICAS
}




\section{PROBLEMAS DEL METODO Y DE LA ENSEÑANZA \\ DE LA METODOLOGIA}

Clemencia Chiappe*

\section{INTRODUCCION}

La Universidad Colombiana quiere girar cada día más alrededor del concepto de investigación. Los programas de postgrado lo hacen como objetivo prioritario y en los currículos de pregrado se constituye cada vez más como parte obligada la formación en investigación.

Lo anterior podría conducirnos a esperar una efervescencia en la producción del andamiaje al servicio de este esfuerzo. Esto es, a la publicación de artículos en temas metodológicos, a la publicación de libros encaminados a la enseñanza de la metodología y, sobre todo, a la discusión metodológica sobre la producción investigativa de las diversas disciplinas o de las diversas áreas-problema.

Sin embargo, este clima no existe todavía en nuestras universidades. Por esta razón la enseñanza de la metodología carece de la agilidad y de la seguridad que caracteriza los debates sobre temas que sí concentran la atención de académicos y/o técnicos.

El aula de clase, para el verdadero profesor, es el momento de ilustrar al alumno sobre la forma como se adelantan uno o varios debates sobre problemas claves de su disciplina, y en nuestro medio la enseñanza de la metodología no responde a estas características. $Y$ es que no es sencillo determinar cuál ha de ser la estructura de un curso de Metodología de la investigación en Ciencias Sociales. No tanto por la sucesión de temas, ya que la guía por excelencia la constituyen los pasos del diseño de investigación. El problema radica en cómo trabajar cada uno de estos pasos. Porque no hay uno sino varios enfoques epistemológico-metodológicos que los conciben de manera diferente. Explicitar esos pasos y mostrar las diferencias de los enfoques metodológicos en la definición de cada uno de ellos constituye una parte tan esencial de la formación científica del investigador como el "aprender haciendo".

Esta es la tarea que nos proponemos guiar a través de una presentación de cuatro temas clásicos de los debates metodológicos. En primer lugar el que se refiere a la Legalidad. Toda hipótesis planteada en una investigación tiene un supuesto anterior que da sentido a su postulación: Suponemos que la realidad no es un caos sino que ocurre de acuerdo a leyes y que podemos conocerlas. Suponemos que los sucesos no son producto del azar sino que responden a una Legalidad, a un conjunto de leyes. Pero hay un segundo supuesto detrás de cada una de esas hipótesis de investigación: Que las leyes son causales, o funcionales o dialécticas, etc. Este es el primero de los cuatro temas a que queremos hacer referencia.

\footnotetext{
* Licenciada en Sociología, Universidad Javeriana, estudios de postgrado en Investigación y tecnología educativa, Universidad Javeriana, M. A. en educación por la Universidad de Berkeley California. Directora del Centro de Investigaciones de la Universidad Pedagógica Nacional.
} 
Viene, en segundo lugar, el problema metodológico de la determinación de la unidad de análisis, que a su vez es la expresión de la disputa clásica por la definición del objeto de estudio de las Ciencias Sociales.

En efecto, en la elaboración del diseño de una investigación —o su equivalente, si se quieren evitar connotaciones unívocas de este término a un exclusivo enfoque metodológico-, la manera como se percibe y define el objeto de las ciencias sociales permea la atención selectiva, la perspectiva del investigador al concretar sus unidades de análisis.

En tercer lugar, como puede deducirse de los dos enunciados anteriores, presentaremos la discusión en torno del concepto de Validez. Nuestros estudiantes, acostumbrados a repetir: "Un indicador es válido cuando mide lo que debe medir" deben ir mucho más allá en el concepto de validez y relacionarlo íntimamente con los problemas de la Legalidad y las opciones en torno de la determinación de la Unidad de Análisis.

Finalmente, como cuarto tema, se trabajará sobre el papel del proceso de Conceptualización y el sentido que se atribuye a ese término en cada uno de los enfoques metodológicos que se han juzgado como básicos para hacer relevante una comparación.

Es nuestro objeto, al desarrollar estos temas, defender la necesidad del estudio teórico-, del problema del método, que servirá de base al "aprender haciendo".

\section{Legalidad e Hipótesis}

El primer gran problema lo constituye la discusión sobre la legalidad de los fenómenos sociales, que es la dimensión epistemológica del problema de la formación de hipótesis en la investigación social. Como decíamos arriba, hay un supuesto detrás de cada una de las investigaciones que repasamos y es el que las leyes que tratamos de hacer evidentes mediante la supuesta prueba de dichas hipótesis son de orden causal, o funcional, o dialécticas o teleológicas. No haremos a continuación una exposición filosófica a la manera de una discusión sobre Bacon, Hume, Kant, o Marx. Nuestra pretensión es más sencilla. Veamos, en primer término, en qué consiste el proceso de formulación de hipótesis desde la concepción empírico-positiva.

Al trabajar dentro de una concepción empírico-positiva el proceso de formulación de hipótesis consiste en expresar una relación funcional (de asociación o interactiva) entre fenómenos. No se afirma una conexión genética sino una asociación externa. Las hipótesis, dentro de esta concepción, expresan una relación y no una conexión, en el sentido de causación.

Su ventaja comparativa reside en precisar las características de esa asociación ya que se trata de determinar el grado en que dos términos de una relación se afectan mutuamente o de manera unidireccional y desembocamos así, necesariamente, en una exigencia de cuantificación para lograr el refinamiento de la predicción. En efecto, esta cuidadosa descripción permite montar enunciados predictivos que pueden alcanzar una enorme exactitud ${ }^{158}$.

\footnotetext{
${ }^{158}$ Sobre este tema son especialmente valiosos los aportes de los coloquios de Hayden sobre Método y Conceptualización Científica. Dos de ellos, en especial, editados por Daniel Lerner: Evidence and Inference, the Free Press of Glencoe, N.Y. 1958 y el segundo: Quantity and Quality, the Free Press of Glencoe, N.Y., 1961. 
Pero no debe olvidarse que esa exactitud depende de que las condiciones que explican el porqué de esa asociación continúen actuando con las mismas características, en los mismos términos. Debe recordarse que la determinación de esas condiciones, o sea, la pregunta por el porqué de la asociación, no tiene cabida en un enfoque que no se pregunta por la causalidad. Por lo tanto, cuando la predicción montada sobre los resultados de la descripción falla, el proceso que se inicia consiste en una nueva descripción de las características de asociación o independencia entre las variables analizadas tal y como se encuentran después de la ocurrencia del evento que hizo fallar la anterior predicción. Así pues, si su ventaja comparativa está en la predicción, su desventaja radica en la incapacidad de dar cuenta de la conexión de los fenómenos, como causa-efecto.

Veamos ahora cómo se da una relación estrecha entre esta opción por un proceso descriptivo-predictivo y las investigaciones aplicadas y operativas ${ }^{159}$.

En primer lugar traigamos a la discusión una definición de los dos términos: Investigación operativa e investigación aplicada. La investigación aplicada busca generar información cuya utilidad es inmediata para plantear alternativas de manipulación de variables con el objeto de alterar las relaciones ya conocidas. En cuanto a la investigación operativa, ella se refiere a la aplicación y utilización de resultados de investigación en el desarrollo de nuevos servicios y acciones.

El enfoque empírico positivo es especialmente útil para la elaboración de investigaciones aplicadas y operativas pues éstas se basan en el conocimiento de predicciones sobre las que se desea actuar, a través de la transformación de la distribución de una población en los valores de una o más variables.

En efecto, una vez conocida una asociación y determinadas con un grado de precisión cuáles son los valores de una variable asociados con los valores de otra, como también las características de variación de una variable en función de la otra es posible diseñar mecanismos - políticas- que alteren ese orden de cosas.

Dejemos hasta aquí, delineadas las características esenciales, nuestra exposición relativa al enfoque empírico positivo, para entrar a discutir el mismo problema de la legalidad desde los postulados histórico-sociales. Nos acercamos a un planteamiento diferente, que incide directamente en la manera de trabajar las hipótesis, y en el ulterior desarrollo del diseño.

Para el enfoque histórico-social, especialmente en la corriente Weberiana, la comprensión de un hecho social se logra por medio de la formulación de hipótesis interpretativas que esperan su verificación empírica.

La comprensión es una forma específica de explicación causal: La determinación de relaciones causa-efecto individuadas o sea, la determinación de las condiciones de posibilidad ligadas a un evento específico.

El interés recae fundamentalmente en la explicación del porqué del tránsito de una situación a otra. Así pues, explicar un fenómeno es comprenderlo. Comprenderlo es formular hipótesis interpretativas, lo que, a su vez, consiste en determinar las condiciones de posibilidad de un evento específico.

\footnotetext{
${ }^{159}$ Abell, Peter.Model building in Sociology. New York, Schocken Books, 1971. Digitalizado por RED ACADEMICA
} 
Hemos dicho que la selección de uno u otro enfoque otorgan un papel diferente a las funciones descriptiva, explicativa y predictiva. La investigación histórico-social utiliza los estudios descriptivos pero ella misma no tiene como finalidad la descripción. Su pretensión es la de explicar. Ahora bien, no se trata de una explicación al servicio de la predicción como extrapolación ya que, al determinar con exhaustividad las condiciones fundamentales para la ocurrencia de un evento particular, el ámbito de extrapolación se restringe en proporción directa a la exhaustividad de la comprensión de las relaciones de causa-efecto específicas del evento.

La ambición de científico-histórico es detectar los factores relevantes, los condicionantes clave para dar cuenta —en el sentido de causar-, el evento en cuestión.

Avancemos ahora hacia nuestro tercer punto referencia en esta comparación. Entramos en el terreno de la perspectiva hermenéutica.

El hermeneuta interpreta el sentido de la acción dentro de un contexto. Y ese sentido o significación se descubre con más frecuencia a través de la pregunta por la finalidad de la acción (orientación teleológica) que a través de la pregunta por sus causas, especialmente cuando se iguala el término causa con variable o fenómeno antecedente de otro, llamado a su vez consecuente. La explicación del hecho social se da en la elucidación de los fines perseguidos, ya que esos fines son los que cargan de sentido la acción.

\section{La determinación de la Unidad de Análisis y la pregunta por el Objeto de las Ciencias Sociales}

El segundo problema lo constituye la determinación de la unidad de análisis. Es decir la explicitación de cuál es el objeto de estudio en las Ciencias Sociales. Pero no nos referimos a la pregunta simple que requiere como respuesta una parcela de los seres vivos, o de algunas de sus actividades, para llamarlas objeto de las Ciencias Sociales.

La pregunta espera una explicación epistemológica sobre cómo se percibe, y por lo tanto, en qué términos puede ser conocida la realidad social. Y la respuesta determinará absolutamente al investigador en la formulación del problema y todos los pasos del diseño.

Así, para el enfoque empírico positivo los objetos de la Ciencia Social son un conjunto de regularidades empíricas. A la base de estas regularidades empíricas se encuentra el dato. Los hechos, objeto de estudio de las Ciencias Sociales, son datos. Y el dato se define por tres características: Observable, externo y dado.

Observable, lo cual descarta, en opinión de esta corriente la "adivinación", la interpretación psicologista. En un segundo momento se liga el criterio de observable con el de medible, y cuantificable.

El concepto de variable y el de indicadores están enteramente montados sobre esta definición y de igual manera el concepto de hipótesis operacionales se deduce de la concepción de legalidad desarrollada en el marco empírico positivo. Como decíamos más arriba, se trata allí de descubrir una relación funcional entre fenómenos, o, siendo más precisos en la terminología, una relación entre variables. 
Veamos cómo se determina la unidad de análisis desde una perspectiva hermenéutica que recuperan hoy día Ricoeur, Gadamer, William Sullivan, Paul Rabinow ${ }^{160}$.

Los objetos de la Ciencia Social se definen como significantes (acciones, relaciones, pero en cuanto que están dotadas de significado). La significación es lo que caracteriza fundamentalmente el comportamiento humano. $Y$ es una significación que se entiende y perfila como tal solo dentro de un conjunto de significaciones más amplio.

La unidad de análisis es el sentido de la acción individual y grupal, las motivaciones internas de individuos y grupos y las transformaciones de sus valores y actitudes en la búsqueda de un fin.

Es evidente el enfrentamiento que tiene que surgir entre hermeneutas y empírico positivistas. Frente a un mismo fenómeno un grupo lo trabaja como el Dato y el otro lo trabaja como el Sentido de la Acción. Uno de ellos se orienta a detectar regularidades empíricas, y descubre asociaciones funcionales entre conjuntos de datos. El otro, por su parte ante la pregunta por el sentido de una acción, se orienta a buscar la finalidad de dicha acción, vale decir, la legalidad teleológica que la explica; o sea que le da sentido.

Dentro de la comparación que estamos desarrollando, corresponde el turno a la perspectiva histórico social y a su concepto de Unidad de Análisis.

La lectura de la introducción de Rossi a Weber ${ }^{161}$ apoya los siguientes comentarios. Lo que distingue a las ciencias histórico-sociales, se deduce de esa presentación, no es la percepción del objeto en una forma Radicalmente diferente, sino el fin con miras al cual es indagado y el método de su elaboración conceptual.

El énfasis está colocado en la formalización de la realidad en términos de categorías: Tal es el caso de categorías como el concepto de la tipología de la autoridad en Weber, o del concepto de modo de producción en Marx.

Las categorías se construyen mediante un procedimiento de abstracción que aísla elementos empíricos y los coordina. De lo anterior resulta un tipo ideal que se diferencia de la realidad empírica y que a la vez debe servir instrumentalmente para la explicación de los fenómenos en su individualidad.

Con base en esas categorías se construyen las hipótesis interpretativas que esperan su verificación empírica.

Hasta aquí hemos construido nuestra argumentación en favor de la discusión sobre el método con base en el desarrollo de dos temas que subyacen y permean el desarrollo del diseño de investigación. El primero de ellos, el concepto de legalidad; el segundo se refiere al concepto de hecho social en lo que afecta la determinación de la unidad de análisis; pasaremos ahora a presentar el tercer tema y es el concepto de validez para cada uno de los enfoques.

\footnotetext{
${ }^{160}$ Véanse por ejemplo las siguientes obras:

Ricoeur, Paul. "Ciencia e Ideología”.Ideasy Valores No. 42-45, 1973-1975.

Gadamer, Hans-Georg. Verdad y Método. Ediciones Sigueme-Salamanca, 1977.

Sullivan William and Rabinow Paul Jntrepretative Social Science, University of California Press, 1978

${ }^{161}$ Weber, Max. Ensayos sobre metodología sociológica. Amorrontu 1975. 


\section{El problema de la validez}

En un enfoque empírico positivo los hechos sociales (datos), se pueden y deben reflejar en los conceptos operacionales. Y las interacciones (relaciones de asociación, dependencia) entre los hechos sociales se pueden y deben reflejar a través, fundamentalmente, de las operaciones estadísticas. Así, pues, la fidelidad se juega en la finalidad y pureza en el proceso de recolección de los datos.

Este concepto de validez es totalmente congruente con sus planteamientos sobre legalidad y determinación de la unidad de análisis y, como se verá a continuación, los otros dos enfoques, también se rigen por un concepto de validez coherente con sus postulados sobre Unidad de Análisis y Legalidad.

En el enfoque hermenéutico el trabajo del investigador es válido cuando se da una coherencia entre el sentido de la acción que los actores le atribuyen y la interpretación que de ese sentido de la acción ofrece el investigador. La validez se reconoce porque se produce un acuerdo; Los actores se reconocen en sus motivaciones a través de la interpretación del investigador y éste reconoce su "conceptualización" en la acción de los actores.

Finalmente, en el enfoque histórico social, explicar un fenómeno es hacer evidentes las causas que lo generaron y por lo tanto es hacer evidentes los caminos de su transformación. Al hablar de legalidad se indicó cómo se "imputa" un acontecimiento a sus causas. Ahora bien, ¿cómo es posible establecer que ellas y no otras han conducido a que sucediese el fenómeno que se quiere explicar?

Se construye un proceso hipotético; se compara el proceso hipotético con el proceso real, con la realidad misma; esa comparación permite establecer la importancia causal de los elementos incluidos como tales y también, como es obvio, permite clarificar la relevancia causal de los elementos excluidos como tales.

Lo que se está tratando de averiguar con base en ese ejercicio es el grado de "adecuación" de las categorías, y la relación postulada entre ellas, y la realidad social en un momento específico. 'El grado de adecuación es la medida de la validez del proceso hipotético.

He aquí, apenas esbozadas, pero conteniendo sus rasgos fundamentales, tres concepciones diferentes sobre validez del conocimiento en Ciencias Sociales: Validez igualada al proceso de Comprobación, en el enfoque empírico-positivo. Validez equiparada a la Coherencia para el enfoque hermenéutico y, finalmente, validez igualada a un proceso específico de Adecuación, en el enfoque histórico-social.

Usualmente una de ellas es la que guía la producción intelectual de un científico social, como también, desde una de ellas se juzga posteriormente el trabajo elaborado.

\section{El significado de la elaboración conceptual}

Hay un abismo entre lo que se acepta como concepto en un estricto acercamiento empirista y el significado de dicho término para el enfoque histórico social. Y distante de ambos también se encuentra la perspectiva hermenéutica. 
Desarrollemos el planteamiento empírico pues, a pesar de que hoy día no se defiende en sus consecuencias más estrictas con relación a la conceptualización, sí ilustra sobre las raíces de términos tales como definición operacional y sobre la discusión sobre relación entre definiciones conceptual y operacional.

Para que la realidad, el dato observado y consignado, hable por ella misma, prescindiendo del potencial subjetivista de la abstracción, se evita hablar de conceptos y se utiliza preferencialmente el término "definición operacional". El ejercicio de presentar definiciones operacionales consiste en "nombrar" constataciones empíricas.

Una vez verificada una relación entre, digamos, dos definiciones operacionales que se han trabajado en una ecuación, se agrega esta confirmación a una cadena de enunciados ya verificados, conformando así el cuerpo de conocimientos.

Tenemos entonces que la conceptualización es un proceso por el cual se nombran relaciones verificadas o por verificar. Es un proceso secundario, subsidiario al esfuerzo primordial de distinguir el dato.

Como dijimos anteriormente, al tratar este tema específico de Conceptualización y buscando "tipificar", acudimos a un planteamiento empirista que está ampliamente revaluado hoy día. Y esta reevaluación ha acercado la definición y el papel de la conceptualización para los empíricos positivistas a la del enfoque histórico social, aunque guarden distancias con relación a otros temas.

Veamos cómo se trabaja la conceptualización en el enfoque histórico social. Realmente lo que distingue a las ciencias socio-históricas no es tanto su discusión con relación al Objeto de las Ciencias Sociales, cuanto el método de su elaboración conceptual. Un énfasis especial se pone en la formalización de la realidad en términos de categorías.

Como habíamos mencionado anteriormente, las categorías se construyen mediante un procedimiento de abstracción que aísla elementos empíricos y los coordina. De lo anterior resulta un tipo ideal que a) se diferencia de la realidad empírica y b) que debe servir instrumentalmente para la explicación de los fenómenos en su individualidad.

Para lograr lo anterior, se constituye en un criterio de comparación al cual es referido el dato empírico.

Finalmente, veamos cómo se percibe este problema de la conceptualización, desde la perspectiva hermenéutica, en sus variantes más antropológicas.

Con este enfoque metodológico se llega a postular una interpretación, que es una teoría cuyo ámbito de validez se circunscribe enteramente al hecho estudiado, en un momento dado, y en un contexto particular. Por lo tanto, lo que de allí surge como concepto tiene, en principio, el mismo ámbito restringido de validez.

El hermeneuta no niega la existencia de esas "teorías" construidas por otros y por él mismo. Pero al entrar a interpretar un objeto no deja que ellas interfieran como preconceptos a los cuales él acomode indebidamente su nuevo objeto de interpretacion.

Me he detenido en el desarrollo de cuatro temas que inciden en el estudio metódico de la realidad social para tres enfoques metodológicos diferentes con el fin de justificar una 
defensa de la formación teórica en Metodología. Cada uno de los temas debe desarrollarse ampliamente; aquí tan solo hemos enmarcado el problema. Así mismo, el tratamiento que hemos dado a los llamados enfoques es bastante introductorio. Queda por trabajar un abanico mucho mayor en el que se despliegan vertientes de cada tino de ellos, de manera altamente interesante al compartir, algunas de esas nuevas líneas, conceptualizaciones de un enfoque, digamos, el hermeneuta, con el histórico social; o de este último combinado con rasgos empírico positivos, etc.

Quisiera, a continuación, recordar una discusión surgida en el primer Simposio Nacional sobre Métodos de Investigación en Educación organizado por la Universidad Externado de Colombia. Es la polémica planteada por Antanas Mockus en su ponencia a dicho Seminario.

En primer término, trataré de resumir la argumentación de Antanas Mockus en contra de las discusiones sobre el método. Destaca como primera tesis el hecho de que asistimos hoy día a la consagración de algunos procedimientos metodológicos como "el instrumento", como "el método" y que, por lo tanto, la validez de la producción científica está subordinada a la posesión y utilización de ese instrumento.

De lo anterior se acostumbra a deducir, precisa Mockus, que el secreto de la producción científica reside en ese instrumento denominado Método Científico. Constituido este último, añade, es preciso darlo a conocer y para ello surgen los cursos especiales de metodología de la investigación social.

Finalmente, llega Mockus a su tesis central al afirmar que "método significa simplemente excluir, en el sentido de descalificar, la producción de otros".

En mi opinión, el doctor Mockus, está rechazando de plano la aseveración de los empírico-positivistas de ser poseedores de un bien precioso: El verdadero camino para conocer la realidad. Uno podría estar de acuerdo con este alegato en contra de una pretensión tan ambiciosa. Sin embargo, quien quiera rechazar un método de investigación particular no debe hacerlo acallando la discusión sobre el método en general, que debe plantear-se a nuestro parecer, en los términos en que se han desarrollado las ideas de estas páginas.

Adherimos a otra afirmación de Mockus cuando dice: "Impugnamos radicalmente toda tesis según la cual la conformidad con un método implique el carácter de "verdad" para sus resultados. En efecto, la medida de la conformidad con un método da cuenta sobre la "coherencia" de una determinada elaboración científica pero no da cuenta de su "verdad".

La lucha para discernir el carácter de verdad de los resultados está dada en la discusión de un enfoque metodológico frente a otros en términos como los que se han esbozado en este artículo al hablar de la legalidad de los fenómenos sociales, o de unidades de análisis, etc.

Sólo una continua reflexión sobre los problemas básicos aquí esbozados habilita al científico social para discutir sobre la posibilidad de verdad de sus hallazgos.

Planteada así la discusión, el método no es un instrumento. Es la concreción de una opinión epistemológica en un momento dado y el motor de la acción del científico. 